\title{
Linking Linear Threshold Units with Quadratic Models of Motion Perception
}

\author{
Humbert Suarez \\ Christof Koch \\ Computation and Neural Systems Program, 216-76, \\ California Institute of Technology, Pasadena, CA 91125 USA
}

Behavioral experiments on insects (Hassenstein and Reichardt 1956; Poggio and Reichardt 1976) as well as psychophysical evidence from human studies (Van Santen and Sperling 1985; Adelson and Bergen 1985; Watson and Ahumada 1985) support the notion that short-range motion perception is mediated by a system with a quadratic type of nonlinearity, as in correlation (Hassenstein and Reichardt 1956), multiplication (Torre and Poggio 1978), or squaring (Adelson and Bergen 1985). However, there is little physiological evidence for quadratic nonlinearities in directionally selective cells. For instance, the response of cortical simple cells to a moving sine grating is half-wave instead of full-wave rectified as it should be for a quadratic nonlinearity (Movshon et al. 1978; Holub and Morton-Gibson 1981) and is linear for low contrast (Holub and MortonGibson 1981). Complex cells have full-wave rectified responses, but are also linear in contrast. Moreover, a detailed theoretical analysis of possible biophysical mechanisms underlying direction selectivity concludes that most do not have quadratic properties except under very limited conditions (Grzywacz and Koch 1987). Thus, it is presently mysterious how a system can show quadratic properties while its individual components do not. We briefly discuss here a simple population encoding scheme offering a possible solution to this problem.

We assume a population of $n$ directionally selective cells whose output is zero if the "somatic potential" $x$ is below a certain threshold $x_{T}$ and whose output is linear for small $x$ above this value $f(x)=\alpha\left\lceil x-x_{T}\right\rceil$ where $\lceil x\rceil=x$ if $x>0$ and 0 otherwise (Fig. 1a). We do not consider further the mechanism generating direction selectivity but will assume that the perceptual response to motion $R$ is given by the sum of the responses of a large number of these neurons. Thus, if the moving stimulus induces the intracellular response $x$ in all $n$ cells, we have

$$
R=\sum_{i=1}^{n} f(x)
$$

Neural Computation 1, 318-320 (1989) (C) 1989 Massachusetts Institute of Technology 

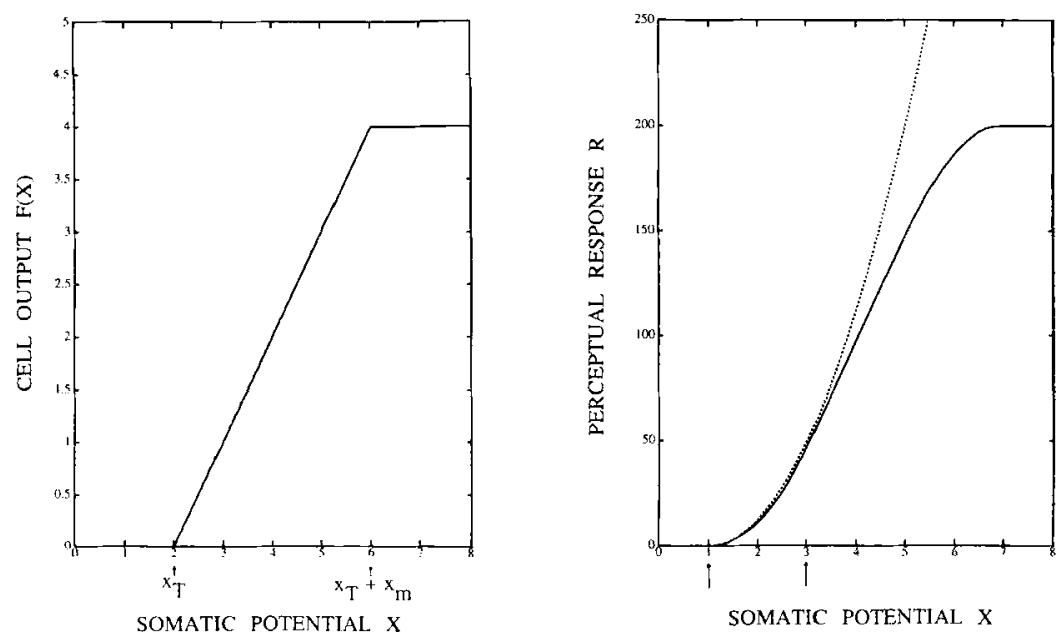

Figure 1: (a) Schematic input-output relationship of a highly idealized directionally selective cell. If the "somatic potential" $x$ is below a threshold $x_{T}$ (here 2 ), the cell remains silent; above this threshold the output of the cell is $\alpha\left(r-x_{T}\right)$, and saturates for $x=x_{1}+r_{m}$. For the simulations described here, we use $x_{m}=4$ and $\alpha=1$. (b) The sum $R$ of the responses for a group of 50 such units with $r_{T}$ uniformly distributed between $x=1$ and $x=3$ (see arrows). $R$ is quadratic for small values of $x$ and saturates for large values. The dashed curve is $12.5(r-1)^{2}$ and corresponds to the expected mean of $R$ for uniformly distributed values of $x$ between 1 and 3 .

If $x_{T}$ is the same for all cells, $R=\operatorname{mr}\left(x-x_{T}\right)$ as long as $r>x_{T}$. However, we will now assume that the threshold varies randomly from cell to cell, let us say distributed uniformly between $x_{T_{1}}$ and $x_{T_{2}}$. If $x$ falls within these values, the function $a\left(x-x_{T_{1}}\right)$ is randomly sampled across this interval prior to summation, and the system then simply computes the area below $f(x)$, similar to Monte-Carlo integration methods. For a sufficiently large value of $n$, we then have that $R$ is proportional to $\left(x-x_{T}\right)^{2}$ [in general, if $f(x)$ is a $m$ th order polynominal, $R$ will be proportional to $\left(r-r_{T_{1}}\right)^{m+1} 1$. Alternatively, if $r_{T}$ were constant for all cells while the "somatic potential" of the neurons was uniformly randomly between $x r_{1}$ and $x$ for a given input, the same quadratic behavior in $x$ would be obtained. In particular, this random variation could be obtained by summing over a population of cells that is broadly tuned for the direction of motion with a certain distribution of preferred directions. 
In all cases, for values of $x$ much higher than $x_{T_{2}}^{\prime}$, the output $R$ will grow linearly since the system will integrate only over a narrow range around $x$. Finally, more realistic neurons saturate at some output value, that is $f(x)=\alpha x_{m}$ for $x>x_{T}+x_{m} . R$ will then saturate also.

The output of this simple system thus approximates a square function for motion in the preferred direction over a range of positive input values (Fig. 1b). By using this averaging technique as well as ON- and OFFrectified "neurons," systems that show quadratic behavior, including fullwave rectification, could in principle be built out of linear threshold units, thereby linking the properties of single cells with the observed behavioral responses. It is rather elegant that this can be accomplished solely by taking into account the random variations in neuronal properties. Note that detailed simulations of more realistic neuronal models are needed to verify the applicability of this mechanism to biological visual systems.

\section{References}

Adelson, E.H., and Bergen, J.R. 1985. Spatiotemporal energy models for the perception of motion. J. Opt. Soc. Am. A2, 284-299.

Grzywacz, N.M., and Koch, C. 1987. Functional properties of models for direction selectivity in retina. Synapse 1, 417-434.

Hassenstein, B., and Reichardt, W. 1956. Systemtheoretische analyse der zeit-, reihenfolgen- und vorzeichenauswertung bei der bewegungsperzeption des rüsselkäfers chlorophanus. Z. Naturforschung 11b, 513-524.

Holub, R.A., and Morton-Gibson, M. 1981. Response of visual cortical neurons of the cat to moving sinusoidal gratings: Response-contrast functions and spatiotemporal interactions. J. Neurophysiol., 46, 1244-1259.

Movshon, J.A., Thompson, I.D., and Tolhurst, D.J. 1978. Spatial summation in the receptive fields of simple cells in the cat's striate cortex. J. Physiol. 283, 53-88.

Poggio, T., and Reichardt, W.E. 1976. Visual control of orientation behavior in the fly: Part II: Towards the underlying neural interactions. Q, Rev. Biophys. 9, 377-438.

Torre, V., and Poggio, T. 1978. A synaptic mechanism possibly underlying directional selectivity to motion. Proc. $R$. Soc. London Ser. B 202, 409-416.

Van Santen, J.P.H., and Sperling, G. 1985. Elaborated Reichardt detectors. J. Opt. Soc. Am. A2, 300-320.

Watson, A.B., and Ahumada, A.J. 1985. Model of human visual-motion sensing. J. Opt. Soc. Am. A2, 322-341.

Received 6 April 1989; accepted 4 July 1989. 